\title{
Density Functional Theory for Molecule-Metal Surface Reactions: When Does the Generalized Gradient Approximation Get It Right, and What to Do If It Does Not
}

Nick Gerrits,* Egidius W. F. Smeets, Stefan Vuckovic, Andrew D. Powell, Katharina Doblhoff-Dier, and Geert-Jan Kroes*

Cite This: J. Phys. Chem. Lett. 2020, 11, 10552-10560

Read Online

ACCESS | Lلll Metrics \& More | 回 Article Recommendations | (1) Supporting Information

ABSTRACT: While density functional theory (DFT) is perhaps the most used electronic structure theory in chemistry, many of its practical aspects remain poorly understood. For instance, DFT at the generalized gradient approximation (GGA) tends to fail miserably at describing gas-phase reaction barriers, while it performs surprisingly well for many molecule-metal surface reactions. GGA-DFT also fails for many systems in the latter category, and up to now it has not been clear when one may expect it to work. We show that GGA-DFT tends to work if the difference between the work function of the metal and the molecule's electron affinity is greater than $\sim 7 \mathrm{eV}$ and to fail if this difference is smaller, with

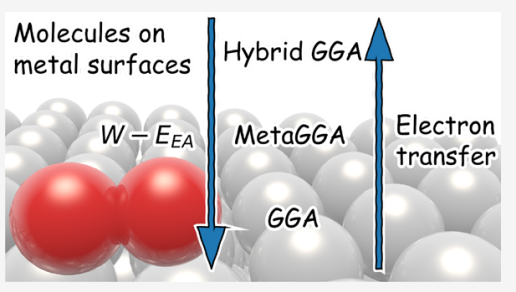
sticking of $\mathrm{O}_{2}$ on $\mathrm{Al}(111)$ being a spectacular example. Using dynamics calculations we show that, for this system, the DFT problem may be solved as done for gas-phase reactions, i.e., by resorting to hybrid functionals, but using screening at long-range to obtain a correct description of the metal. Our results suggest the GGA error in the $\mathrm{O}_{2}+\mathrm{Al}(111)$ barrier height to be functional driven. Our results also suggest the possibility to compute potential energy surfaces for the difficult-to-treat systems with computationally cheap nonself-consistent calculations in which a hybrid functional is applied to a GGA density.

$\mathrm{K}^{\mathrm{s}-2}$ ohn-Sham DFT has become very popular and is now being used in more than 30000 papers per year. Nevertheless, much of the theory remains not yet well understood, even concerning much of its practical aspects. One surprising practical aspect is that density functionals (DFs) at the generalized gradient approximation (GGA) are quite accurate for barriers for dissociative chemisorption (DC) reactions on metal surfaces. In contrast, semilocal functionals tend to systematically underestimate reaction barrier heights of gas-phase reactions, ${ }^{1,2}$ and it has been assumed that this overestimation should carry over to surface reactions in general. ${ }^{3}$ Nonetheless, a semiempirical version of DFT, the specific reaction parameter (SRP) approach to DFT, has achieved chemically accurate descriptions of sticking in many molecule-metal surface systems by using functionals in which the exchange part is a weighted average of GGA exchange functionals. ${ }^{4-12}$ Here, often (the exchange part of) the $\mathrm{PBE}^{13}$ (or PW91 ${ }^{14}$ ) and the RPBE ${ }^{15}$ DFs are used, with PBE and PW91 yielding too low, and RPBE too high barriers. ${ }^{4,6}$ Dynamics studies using nonempirical GGA DFs have semiquantitatively described many experiments on DC on metals. ${ }^{16-20}$ Perhaps even more surprisingly, a recent comparison of DFT results for a database of molecule-metal surface reactions based on experiments and SRP-DFT (SBH10) suggested a better performance for a GGAexchange-based DF (i.e., BEEF-vdW ${ }^{21}$ ) than for the metaGGA and screened hybrid representative examples that were tested. $^{22}$
In spite of the above, also many molecule-metal surface systems exist for which SRP-DFs based on GGA exchange DFs do not work because even the most repulsive GGA exchange DFs still obeying the uniform electron gas limit (such as RPBE, a nonempirical functional which describes adsorption on metals with quite high accuracy, but already performs rather poorly at describing solid state properties ${ }^{21,23}$ ) are too reactive compared to experiment, even when simulating the effect of energy dissipation to electron-hole pairs and surface phonons. ${ }^{24-29}$ As a result, the barrier height cannot be "tweaked" to a good value by mixing exchange DFs yielding too high barriers (such as RPBE) and too low barriers (as often the case with PBE or PW91). That is a pity, as a DC transition state (TS) on a metal surface is often the rate-controlling state in industrially important heterogeneously catalyzed processes $^{30-32}$ like ammonia production ${ }^{33}$ and steam reforming. ${ }^{34}$ A further problem is that it is not clear which property of the system determines whether GGA-DFT may yield a reliable barrier height. Reasons to explain the GGA-DFT failure for individual systems have, however, been cited, with an often

Received: August 11, 2020

Accepted: November 20, 2020

Published: December 9, 2020 


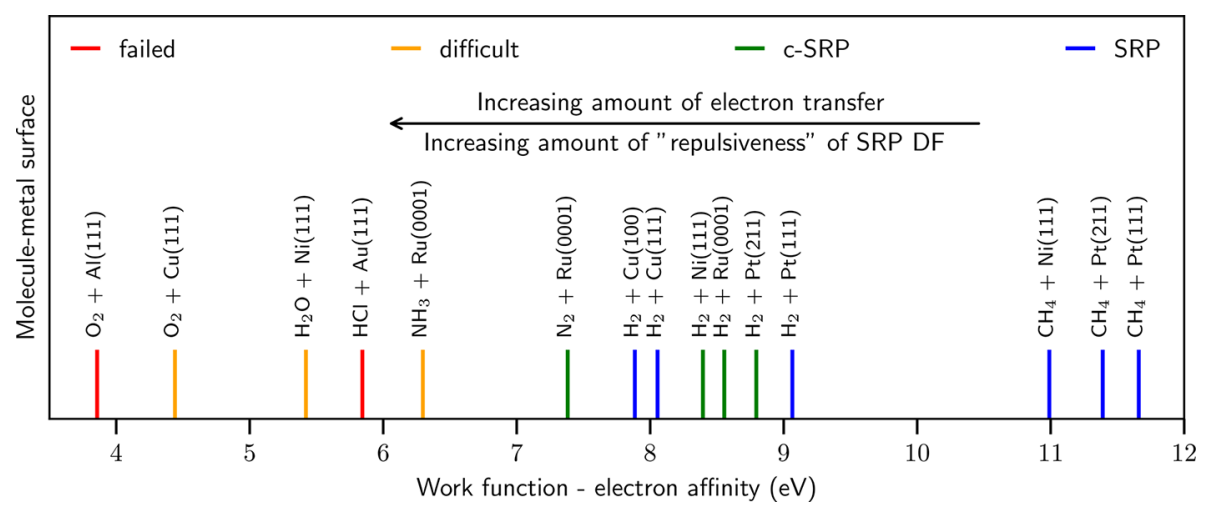

Figure 1. Correlation between the difference of the work function of the metal surface and the electron affinity of the molecule $(\mathrm{eV})$ with the ability of GGA exchange-based DFs to accurately describe barrier heights to DC in the systems described. Red, orange, green, and blue indicate whether efforts to develop an SRP DF based on GGA exchange for a molecule-metal surface reaction have failed, proven difficult, yielded a candidate SRP DF, or yielded an SRP DF, respectively. The values of the work functions (Table S1) and electron affinities (Table S2) are obtained as described in Section S1.5 of the Supporting Information.

cited reason being that electron transfer occurs from the metal surface to the molecule. $^{35,36}$

In this connection, often reference is made to the following explanation for why GGA-DFT fails at describing gas-phase reactions: the GGA's tendency to underestimate gas-phase barrier heights is caused by the electrons delocalizing over additional atoms at the TS, artificially lowering the GGA energy of the TS relative to that of the reactants. ${ }^{2,37,38}$ The delocalization error finds its origin in what has been called "the lack of derivative discontinuities of semi-local functionals" or alternatively the violation of the Perdew-Parr-Levy-Balduz condition ${ }^{39}$ by these functionals. According to Yang and coworkers $^{40}$ the delocalization error is similar but not equal to the self-interaction error (SIE), ${ }^{41}$ which is also often invoked to explain the underestimation of gas-phase reaction barrier heights. However, the above does not yet explain why GGADFT does work for many molecule-metal surface reactions. Also, a property of the system on the basis of which one could straightforwardly predict whether GGA-DFT should, at least in principle, work is still missing. Fortunately, the "charge transfer" explanation does suggest such a criterion, as we will now show.

Here, we will show that a single property of molecule-metal surface systems exists on the basis of which one can decide whether it should be possible to find a GGA functional with which one can describe the barrier to dissociative chemisorption with chemical accuracy. This allows one to define a single corresponding criterion stating whether GGA functionals should be able to deliver chemical accuracy for the corresponding "easy-to-handle" reactions. We also show that the solution applicable to the problem that gas-phase reaction barriers are not well-described with GGA functionals (i.e., resorting to hybrid functionals) also works for a prominent example of "difficult" surface reactions, i.e., that of $\mathrm{O}_{2}+$ $\mathrm{Al}(111)$. Our findings suggest the possibility of extending SRPDFT to the full range of dissociative chemisorption reactions on metals. Such an extension should enable the development of databases for such reactions ${ }^{42}$ without bias to specific rungs of functionals, similar to databases that already exist for gasphase reactions. ${ }^{1,2}$

Obviously, one would expect the tendency of a system to display charge transfer to correlate with the difference ( $W-$ $E_{\text {ea }}$ ) between the work function $W$ of the metal and the electron affinity $E_{\mathrm{ea}}$ of the molecule. Systematic evidence is collected in Figure 1, where we show how the ability to accurately describe the measured reactivity of molecule-metal surface systems with DFs containing GGA exchange correlates with $W-E_{\text {ea }}$. Figure 1 shows that it has been possible to achieve chemical accuracy in descriptions of sticking experiments with GGA-exchange-based SRP-DFs ${ }^{4,5,7-9}$ (in blue) and candidate SRP-DFs ${ }^{6,10-12}$ (in green) if $W-E_{\text {ea }}$ exceeds $7 \mathrm{eV}$. (Candidate SRP-DFs describe a sticking experiment on a specific system with chemical accuracy, but their validity has not (yet) been confirmed through comparison with another experiment on the same system.) However, for systems with $W$ $-E_{\text {ea }}<7 \mathrm{eV}$ DFs with repulsive RPBE exchange either overestimate sticking notoriously ${ }^{24,25,28,43-45}$ (red), or they show a suspect performance ${ }^{26,27,29,46}$ (orange). In Figure 1 we also observe a trend that when the difference between $W$ and $E_{\text {ea }}$ decreases, so that one might also expect the amount of electron transfer to increase, the SRP or GGA DF needs to be made increasingly repulsive to describe the system's reactivity with chemical accuracy. For example, $\mathrm{CH}_{4}+\mathrm{Ni}(111)^{47}$ and $\mathrm{Pt}(111)^{48}$ are quite well-described with the attractive $\mathrm{PBE}$ functional, ${ }^{13}$ and this is also true for $\mathrm{H}_{2}+\mathrm{Pt}(111)^{49}$ (here the PW $91^{14}$ functional was used, which may be considered the predecessor to PBE, which was designed to replace it ${ }^{13}$ ) and $\mathrm{Ru}(0001)^{6}$. On the other hand, the SRP DF for $\mathrm{H}_{2}+$ $\mathrm{Cu}(111)^{4}$ and $\mathrm{Cu}(100)^{5}$ needs to contain about $50 \% \mathrm{RPBE}$ exchange, and a good description of $\mathrm{N}_{2}+\mathrm{Ru}(0001)$ was recently obtained with the RPBE DF. ${ }^{10}$

A caveat with the above comparison between dynamics based on DFT and experiment is that the difference $\left(W-E_{\mathrm{ea}}\right)$ has also been correlated with the extent to which (reactive) scattering in a system may be affected by energy dissipation through electron-hole pair excitation. ${ }^{50}$ Here, the reasoning used could be that (electronically adiabatic) dynamics simulations using repulsive RPBE exchange might overestimate the DC probability because the dissipation of the molecule's incident kinetic energy to electron-hole pairs is not modeled. However, in this type of analysis evidence for strongly nonadiabatic molecule-metal surface scattering comes mostly from experiments on vibrationally inelastic scattering ${ }^{51,52}$ and scattering of $\mathrm{H}$ atoms from metal surfaces, ${ }^{53}$ whereas dynamics calculations only suggested small effects of electron-hole pair excitation in some of the hard to model systems in Figure 1, i.e., $\mathrm{D}_{2} \mathrm{O}+\mathrm{Ni}(111)^{54}$ and $\mathrm{HCl}+\mathrm{Au}(111) .^{28}$ More definite evidence that electronically nonadiabatic effects are most likely 
not the explanation for the trend observed in Figure 1 comes from a direct comparison between barrier heights obtained with first-principles and RPBE calculations. Barrier heights obtained with diffusion Monte Carlo (DMC) $)^{55}$ and DFT using the RPBE and PBE DFs are compared for three $\mathrm{H}_{2}+$ metal surface systems in Table 1 . For $\mathrm{H}_{2}+\mathrm{Cu}(111)^{56}$ and $\mathrm{Al}(110)^{57}$

Table 1. Barrier Heights $(\mathrm{kJ} / \mathrm{mol})$ Computed with DMC Compared with Values Calculated with the RPBE and PBE (or PW91 ${ }^{*}$ ) DFs of DFT for Three Different $\mathrm{H}_{2}+$ Metal Surface Systems ${ }^{a}$

\begin{tabular}{ccccl}
$\begin{array}{c}\text { molecule-metal } \\
\text { surface }\end{array}$ & $E_{\mathrm{b}}^{\mathrm{QMC}}$ & $E_{\mathrm{b}}^{\mathrm{RPBE}}$ & $E_{\mathrm{b}}^{\mathrm{PBE}}$ & \multicolumn{1}{c}{$W-E_{\mathrm{ea}}$} \\
$\mathrm{H}_{2}+\mathrm{Cu}(111)$ & $54.4 \pm 4.2^{56}$ & $79.1^{4}$ & $46.9^{\ddagger 4}$ & 8.06 \\
$\mathrm{H}_{2}+\mathrm{Al}(110)$ & $105.0 \pm 0.8$ & 100.4 & 79.9 & 7.39 \\
$\mathrm{H}_{2}+\mathrm{Mg}(0001)$ & $113.8 \pm 2.9^{58}$ & $103.3^{58}$ & $84.9^{58}$ & $6.92^{b}, 6.82^{c}$
\end{tabular}

${ }^{a}$ Also shown are the differences $W-E_{\text {ea }}(\mathrm{eV})$ between the work function of the metal and the electron affinity of $\mathrm{H}_{2}$ as computed at the semi-empirical composite G4 level of theory. ${ }^{59}{ }^{b}$ Calculated for $\mathrm{Mg}(0001)$ with PBE-DFT. ${ }^{60}{ }^{c}$ Measured for a thin layer of $\mathrm{Mg} .{ }^{61}$

GGA DFT is able to reproduce the DMC barrier height, and $W-E_{\mathrm{ea}}>7 \mathrm{eV}$. In contrast, the RPBE DF underestimates the DMC barrier height for $\mathrm{H}_{2}+\mathrm{Mg}(0001)^{58}\left(W-E_{\text {ea }}<7 \mathrm{eV}\right)$. Note that for $\mathrm{H}_{2}+\mathrm{Cu}(111) \mathrm{DMC}$ was shown ${ }^{56}$ to be able to reproduce the best estimate of the barrier height to within 6.7 $\pm 4.2 \mathrm{~kJ} / \mathrm{mol}$. Below we will attempt an explanation of the success of exchange-correlation functionals containing semilocal exchange at describing reaction barrier heights for molecule-metal surface systems with $W-E_{\text {ea }}>7 \mathrm{eV}$ and of their failure for systems with $W-E_{\mathrm{ea}}<7 \mathrm{eV}$. However, first we will investigate whether and how the problem can be fixed for one of the "difficult" systems described above.

The standard way of dealing with errors in barriers for gasphase reactions is to ascend the DFT ladder to higher functionals, i.e., to use meta-GGA or hybrid DFs. We will now show that this route can also be successful when dealing with molecule-metal surface reactions. One clear example where DFT tends to overestimate the reactivity is for useful benchmark systems ${ }^{42}$ of activated dissociation of $\mathrm{O}_{2}$ on metal surfaces ${ }^{24,26}$ (see also the Supporting Information), with $\mathrm{O}_{2}+\mathrm{Al}(111)$ being an infamous example. ${ }^{24}$ Molecular dynamics (MD) simulations employing GGA DFs predict a nonactivated reaction, ${ }^{24,43}$ whereas experiments show that the reaction is activated ${ }^{62,64}$ (see Figure $2 a$ ). To date, of the MD simulations that use a GGA DF (or a semiempirical potential energy surface (PES) based on a GGA DF), ${ }^{24,43,65}$ only calculations that treat the $\mathrm{O}_{2}-$ metal system nonadiabatically have been shown to yield sticking probabilities in semiquantitative agreement with experiment. On the other hand, electronically adiabatic simulations that use embedded correlated wave function (ECW) theory for the PES have also yielded quite good agreement with experiment ${ }^{63}$ (see Figure 2a). The latter results led Carter and co-workers to suggest that modeling electronically nonadiabatic effects should not be necessary for $\mathrm{O}_{2}+\mathrm{Al}(111) .{ }^{36}$ Specifically, electronic structure calculations based not only on ECW theory but also on hybrid DFT yield adiabatic barriers, ${ }^{36,66-71}$ suggesting that an electronically adiabatic approach could well be valid, but that the way the electronic structure is treated is crucial. However, drawbacks of the ECW method are that it is expensive to use and that it is hard to converge the molecule-

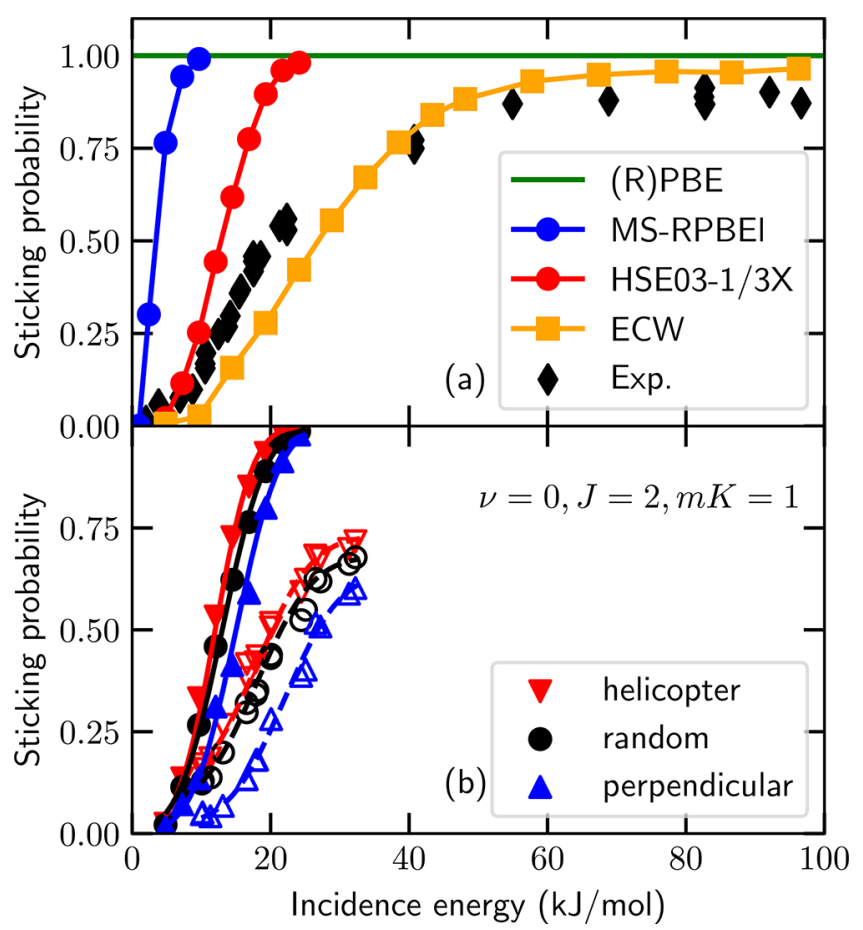

Figure 2. (a) Sticking probability of $\mathrm{O}_{2}$ on $\mathrm{Al}(111)$ as a function of translational energy for normal incidence. The green line indicates results obtained with the (R)PBE DF, ${ }^{24,43}$ whereas the blue and red circles indicate QCT results obtained here with the MS-RPBEl and HSE03-1/3X DFs, respectively. The black diamonds and orange squares indicate experimental results ${ }^{62}$ and QCT results obtained with an ECW PES, ${ }^{63}$ respectively. (b) Sticking probability of $\mathrm{O}_{2}(\nu=0, J=$ 2, $K=1$ ) on $\mathrm{Al}(111)$ for the helicopter (red downward pointing triangle), random (black circle), and perpendicular (blue upward pointing triangle) orientations. The solid and open symbols indicate the computed (HSE03-1/3X) and experimental ${ }^{64}$ results, respectively. The lines merely guide the eye.

surface interaction energy with respect to the size of the embedded cluster. ${ }^{36}$ Consequently, Yin et al. ${ }^{63}$ had to base their PES on a limited amount of points, which forced them to adopt a fitting method that is of only medium accuracy (i.e., a flexible periodic London-Eyring-Polanyi-Sato (FPLEPS) function $^{72}$ ) to represent their ECW data. Additionally, it is not so clear how the ECW method could be used in an SRP approach, whereas this is rather obvious for DFT.

We have investigated the dissociative chemisorption of $\mathrm{O}_{2}$ on a static $\mathrm{Al}(111)$ surface with the quasi-classical trajectory $(\mathrm{QCT})^{73}$ method using PESs based on DFs that go beyond the standard GGA. Like Carter and co-workers, we make the static surface approximation and the Born-Oppenheimer approximation, thereby neglecting nonadiabatic effects related to the spin- and charge-states of $\mathrm{O}_{2}$ and to electron-hole pair excitation. For our calculations, we have developed 6D PESs with the meta-GGA MS-RPBEl $\mathrm{DF}^{74}$ and the screened hybrid HSE03-1/3X DF. ${ }^{75,76}$ The MS-RPBEl DF ${ }^{74}$ is able to describe both covalent and metallic interactions accurately by relying on a switching function dependent on the kinetic energy density. ${ }^{77}$ With this DF the SIE is reduced by ensuring that the exact energy of the free hydrogen atom is reproduced. For the metallic density regime the low-order gradient expansion of the exchange energy of the homogeneous electron gas is reproduced, which ensures a good description of the metal. The HSE03-1/3X DF is based on the HSE03 DF, ${ }^{75,76}$ with the 
Table 2. Barrier Location $\left(Z_{\mathrm{O}_{2}}, \AA\right)$ and Height $\left(E_{\mathrm{b}}, \mathrm{kJ} / \mathrm{mol}\right)$ of $\mathrm{O}_{2}$ on $\mathrm{Al}(111)$ Obtained from Raw ECW Data ${ }^{63}$ and the MSRPBEl and HSE03-1/3X CRP PESs Generated in This Work ${ }^{a}$

\begin{tabular}{|c|c|c|c|c|c|c|c|}
\hline \multirow[b]{2}{*}{ site } & \multirow[b]{2}{*}{ orientation } & \multicolumn{3}{|c|}{$Z_{\mathrm{O}_{2}}(\AA)$} & \multicolumn{3}{|c|}{$E_{\mathrm{b}}(\mathrm{kJ} / \mathrm{mol})$} \\
\hline & & ECW & MS-RPBEl & HSE03-1/3X & ECW & MS-RPBEl & HSE03-1/3X \\
\hline \multirow[t]{4}{*}{ fcc } & $/ / 1$ & 1.9 & 3.0 & 2.6 & 60 & $3.7(3.7)$ & $12.3(12.9)$ \\
\hline & $/ / 2$ & 2.2 & 3.0 & 2.7 & 43 & $3.4(2.0)$ & $11.4(10.7)$ \\
\hline & $/ / 3$ & 2.4 & 3.0 & 2.6 & 18 & $3.7(1.0)$ & $12.3(10.0)$ \\
\hline & $\perp$ & 1.9 & 3.0 & 2.8 & 41 & $8.8(7.4)$ & $26.9(26.7)$ \\
\hline \multirow[t]{2}{*}{ top } & // & 2.6 & 2.8 & 2.6 & 64 & $9.3(7.8)$ & $22.2(21.3)$ \\
\hline & $\perp$ & 2.8 & 3.1 & 2.8 & 64 & $12.0(10.6)$ & $26.8(26.8)$ \\
\hline \multirow[t]{2}{*}{ bridge } & // & 2.4 & 2.7 & 2.5 & 54 & $14.3(12.9)$ & $29.4(28.8)$ \\
\hline & $\perp$ & 2.7 & 3.2 & 2.9 & 43 & $7.3(5.9)$ & $19.4(15.7)$ \\
\hline
\end{tabular}

${ }^{a}$ The nomenclature of the different orientations is taken from ref 63 . The zero-point energy corrected barriers are provided in the brackets.

only difference being the maximum fraction of exact exchange $\alpha$, which may be viewed as a semiempirical parameter. Specifically, we use $\alpha=1 / 3$ instead of $\alpha=1 / 4$, because Cortona and co-workers ${ }^{78,79}$ have shown that increasing $\alpha$ to $1 / 3$ is also valid according to the standard DFT adiabatic connection, ${ }^{78}$ and that the increase from $1 / 4$ as in PBE0 to $1 / 3$ improves the description of gas-phase reaction barriers. ${ }^{79}$ More generally, it is known that hybrid functionals with high fractions of exact exchange tend to perform better at describing reaction barrier heights. ${ }^{2}$ Importantly, the HSE03 DF uses screened exact exchange so that a correct description of the long-range Coulomb interaction is recovered for the metal, and screened hybrid DFT is an order of magnitude cheaper than global hybrid DFT for metals. ${ }^{80-85}$ Both PESs have been constructed by interpolating the DFT data with the corrugation reducing procedure (CRP), ${ }^{86,87}$ of which we summarize the computational details in Sections S1.2 and S1.3. Two-dimensional cuts through the computed PESs are shown for the minimum barrier geometry found in Figures S1 and S2. For the MD, the QCT method ${ }^{73,88}$ is employed, which is also summarized in Section S1.4. The sticking probabilities are computed as described in the Supporting Information of ref 63.

Table 2 compares several barrier heights and locations (i.e., the distance to the surface $Z_{\mathrm{O}_{2}}$ ) obtained with ECW theory, ${ }^{63}$ the MS-RPBEl DF, and the HSE03-1/3X DF. The DFs yield similar trends as ECW theory, except for the geometries with parallel orientations at the fcc site. Generally, both the barrier heights and the anisotropy are lower with the DFs than with ECW theory, and the discrepancies between MS-RPBEl and ECW theory are greater than between HSE03-1/3X and ECW theory. More specifically, among the barrier heights listed the minimum barriers are $3.4,11.4$, and $18 \mathrm{~kJ} / \mathrm{mol}$ for the metaGGA DF, the hybrid GGA DF, and ECW theory, respectively. Furthermore, the difference between the smallest and largest barrier heights listed, which measures how the barrier height varies with impact site (energetic corrugation) and molecular orientation (anisotropy) increases in the same order as 10.9, 17.1 , and $46 \mathrm{~kJ} / \mathrm{mol}$, respectively. Qualitative predictions regarding the dependence of the sticking (or dissociative chemisorption) probability $\left(S_{0}\right)$ on incidence energy $\left(E_{\mathrm{i}}\right)$ can then be made with the hole model. ${ }^{89}$ This model holds that $S_{0}\left(E_{\mathrm{i}}\right)$ is proportional to the fraction of impact sites and orientations for which $E_{\mathrm{i}}$ exceeds the barrier height. The hole model then predicts that the meta-GGA DF yields the lowest energy threshold in the $S_{0}$ curve, while the highest threshold should be found for the ECW results, with the HSE03-1/3X threshold being intermediate. Additionally, based on the barrier height variation the model predicts the steepest $S_{0}$ curve for the meta-GGA DF and the slowest rising curve for the ECW method.

The computed $S_{0}\left(E_{\mathrm{i}}\right)$ curves shown in Figure 2a display the behavior predicted by the hole model on the basis of the barrier heights shown in Table 2. We focus on the overall sticking of $\mathrm{O}_{2}$ on $\mathrm{Al}(111)$ even though this includes contributions from both dissociative chemisorption and abstraction. $^{90}$ The MS-RPBEl DF yields a qualitative improvement over the (R)PBE GGA DF by describing the reaction as activated, but with its low-energy threshold and steep rise with $E_{\mathrm{i}}$ it still overestimates the reactivity considerably. The HSE03$1 / 3 \mathrm{X}$ DF yields considerably better agreement with experiment. The comparison suggests that the minimum barrier height is well-described with the HSE03-1/3X DF, although the slope of the sticking curve is still too steep. In line with the above the latter observation suggests that the anisotropy of the barrier height in $\theta$ and $\phi$ is underestimated and that the energetic corrugation of the barrier height may be too low. The too low anisotropy of the HSE03-1/3X PES also explains why the rotational alignment dependence of the sticking probability is underestimated, even though the calculations qualitatively reproduce the dependence found experimentally (see Figure $2 \mathrm{~b}$ and Section S2.5 for further discussion). ECW theory yields the best overall agreement with experiment, especially regarding the slope. However, the reaction threshold appears to be better described with the tested hybrid functional, suggesting that the HSE03-1/3X minimum barrier height is more accurate than the $\mathrm{ECW}$ value. We also note that the ECW results are based on an approximately fitted FPLEPS PES whereas our dynamics results are based on PESs accurately interpolating the DFT data with the CRP. Furthermore, the agreement of the ECW results with experiment at low incidence energies may have been improved artificially by simulating the reaction of nonrotating $\mathrm{O}_{2}$ instead of using the appropriate rotational distribution ${ }^{63}$ (see Figure S4 and Section S2.4).

The above conclusions are valid provided that the sticking is not much affected by electron-hole pair (ehp) excitation and surface atom motion, the effects of which could lower the energy threshold and the steepness of $S_{0}\left(E_{\mathrm{i}}\right)$. We believe these possible effects to be unimportant for the following reasons: First, experimental results indicate that the surface temperature does not influence $S_{0}{ }^{62}$ Second, according to the local density friction approximation, the probability to excite ehps will be high only if the dynamics sample high electron densities. 
However, for $\mathrm{O}_{2}+\mathrm{Al}(111)$, the barrier is early (far away from the surface), so that the electron density sampled by $\mathrm{O}_{2}$ before it encounters a barrier is low. Third, the location of the barrier far from the surface also suggests small electronic and mechanical couplings ${ }^{91,92}$ with surface atom motion, i.e., the barrier height and location should not vary much with the motion of the nearest surface atom. According to the lattice relaxation sudden model, ${ }^{91,92}$ surface atom motion should then not much affect $S_{0}$.

We suggest that the reasonably good description of the minimum barrier obtained with the HSE03-1/3X DF is due to the reduction of the delocalization error (or the SIE) as a fraction of exact exchange energy is included. ${ }^{38,93-96}$ Furthermore, the HSE03-1/3X DF also qualitatively reproduces experimental alignment and incidence angle-dependent sticking probabilities (see Sections S2.5 and S2.6, respectively). We conclude that the HSE03-1/3X DF already gives a reasonable description of the reaction of $\mathrm{O}_{2}$ on $\mathrm{Al}(111)$, suggesting that an accurate SRP-DF for this system can be developed on the basis of screened hybrid DFs. As further discussed in Section S3.2, we expect that a functional better describing the sticking in $\mathrm{O}_{2}+\mathrm{Al}(111)$ would contain a correlation function approximately describing the van der Waals dispersion interaction, ${ }^{97-99}$ while $\alpha$ should then probably be increased in the hybrid functional.

We now come to a tentative explanation of why semilocal DFT may be quite accurate for barriers to dissociative chemisorption on metals if $\left(W-E_{\mathrm{ea}}\right)>7 \mathrm{eV}$. One of us along with others has recently suggested ${ }^{7}$ a possible reason for this success in terms of the delocalization error of Yang and coworkers. $^{37,38,40}$ The explanation is necessarily of a hand waving nature, as delocalization errors, like self-interaction errors, ${ }^{95,100}$ are usually hard to quantify for most systems of practical interest. The explanation takes its cue from the explanation of the GGA's tendency to underestimate gas-phase barrier heights given in the introductory paragraphs of our Letter. The explanation of the GGA's success in describing moleculemetal surface reactions that may be applied if $\left(W-E_{\mathrm{ea}}\right)>7 \mathrm{eV}$ runs as follows: The electrons coming from the molecule to form new bonds are too delocalized at the TS, and this leads to a delocalization error; ${ }^{40}$ but the electrons coming from the metal were already quite delocalized in the metal, and they become more localized at the TS, leading to a localization error. If electron transfer does not occur too easily (i.e., $W-$ $E_{\text {ea }}>7 \mathrm{eV}$ ), then apparently a cancellation of errors occurs, allowing one to tweak the barrier by mixing GGA-exchangebased DFs. Presumably, the cancellation effect disappears once $\left(W-E_{\mathrm{ea}}\right)<7 \mathrm{eV}$ and the electrons coming from the metal start to spill over to the molecule because of charge transfer. The diffuse charge distribution on the negatively charged molecule upsets the balance leading to too much electron delocalization and therefore an underestimation of the TS energy. The explanation finds quantitative support in the high Bader charges found on the molecules in the TSs of the difficult systems (see Table S8 and Section S2.3).

A remaining question concerns the origin of the delocalization error that plagues the accuracy of GGA barrier heights for systems with $\left(W-E_{\mathrm{ea}}\right)<7 \mathrm{eV}$, which we address here for $\mathrm{O}_{2}+\mathrm{Al}(111)$. There are two possibilities. First, it is possible that the change from reactants to the TS (at which the true electron density will usually be more delocalized) by itself drives the underestimation of the barrier height, i.e., that the error in the barrier height is functional driven. ${ }^{101,102}$ Second, the error may also come about, or be further increased, because semilocal functionals yield self-consistent electron densities at the TS that differ from the true densities to the extent that density driven errors, which may result from over-delocalization, result. ${ }^{101,102}$ The question is relevant: even for stretched $\mathrm{H}_{2}{ }^{+}$, which is a prototypical case for delocalization error, ${ }^{38}$ the error of GGAs is still predominantly functional-driven. ${ }^{103}$ In other words, the self-consistent results are not much improved when GGAs are evaluated on the exact density of stretched $\mathrm{H}_{2}{ }^{+}$. 103

To estimate the RPBE's density-driven error (DDE) for the barrier height of $\mathrm{O}_{2}+\mathrm{Al}(111)$, we evaluate $\mathrm{RPBE}$ on the presumably more accurate HSE03-1/3X DF electron density, i.e., RPBE@HSE03-1/3X. Interestingly, this yields almost the same barrier height as the self-consistent RPBE (see Figure 3).

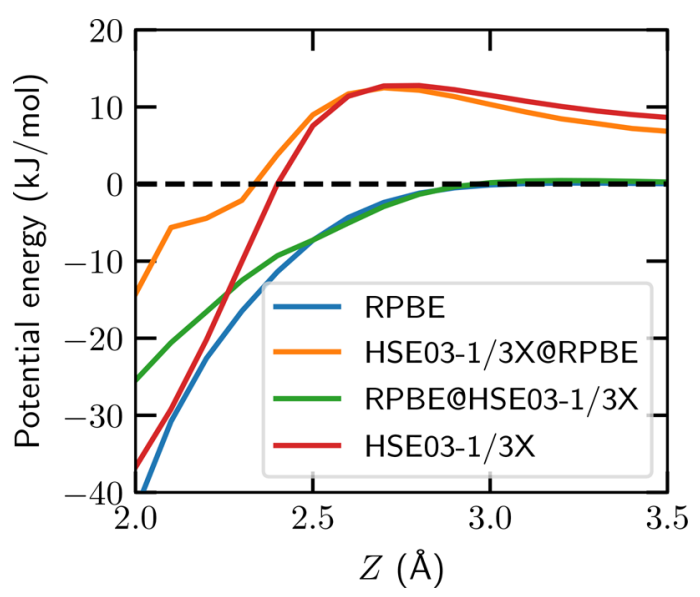

Figure 3. Potential energy of $\mathrm{O}_{2}+\mathrm{Al}(111)$ as a function of $Z_{\mathrm{O}_{2}}$ for $r$ $=1.25 \AA$ and the $\mathrm{fcc} / / 3$ configuration computed self-consistently and nonself-consistently with the RPBE and HSE03-1/3X functionals applied to self-consistent RPBE and HSE03-1/3X electron densities.

This shows, at least for the early barrier system investigated here, that the error of RPBE is functional-driven and not density-driven. At the same time, to a good approximation, the HSE03-1/3X@RPBE energy equals the HSE03-1/3X energy in the entrance and barrier region of the $\mathrm{O}_{2}+\mathrm{Al}(111) \mathrm{PES}$ (see Figure 3 and Table S6). Our results therefore show that the RPBE error in the barrier height is not due to "spuriously easy charge transfer" as suggested in ref 36 because this should be reflected in the RPBE density to which the HSE03-1/3X is applied in HSE03-1/3X@RPBE. Our result that HSE03-1/ 3X@RPBE yields similar energies as HSE03-1/3X in the entrance and barrier regions of the $\mathrm{O}_{2}+\mathrm{Al}(111)$ PES suggests that the HSE03-1/3X@RPBE approach might accurately describe the sticking probability for this and other DC on metal systems. This is a potentially useful finding because using the HSE03-1/3X@RPBE approach to compute energies is roughly an order of magnitude less expensive than using the HSE03-1/3X DF, ${ }^{104}$ with obvious implications for dynamics studies based on hybrid density functionals. While the DDE has a small contribution to the total RPBE error around the TS, its DDE becomes large when the molecule gets closer to the surface (see Figure 3 and Section S2.2). This finding is consistent with the results of Perdew and co-workers for molecular adsorption of $\mathrm{CO}$ on $\mathrm{Pt}(111)$, who also find that the DDE of semilocal DFT is large when the molecule is close to the surface. ${ }^{105}$ 
In conclusion, comparison of experiment and DFT-based theory, and of DMC and RPBE DFT calculations for sticking of molecules on metal surfaces suggests that GGA-DFT starts to fail for molecule-metal surface reaction barriers when $W-$ $E_{\mathrm{ea}}<7 \mathrm{eV}$. Our results obtained for $\mathrm{O}_{2}+\mathrm{Al}(111)$ with the MSRPBEl DF suggest that meta-GGA DFs of the "made simple (MS)" kind can slightly remedy the SIE problem, but not sufficiently for this system. Screened hybrid DFs like HSE031/3X, and its much cheaper HSE03-1/3X@RPBE alternative, offer a considerably improved description of $\mathrm{O}_{2}+\mathrm{Al}(111)$ in that they yield sticking probabilities in semiquantitative agreement with experiment, thus offering more promise, as also implied by single-point calculations performed at reaction barrier geometries of two other difficult systems (see Table S7 and Section S2.2). The HSE03-1/3X DF gives a good description of the reaction threshold but still overestimates the slope of the sticking probability curve for $\mathrm{O}_{2}+\mathrm{Al}(111)$. Our results suggest that SRP-DFs can be built on the basis of screened hybrid exchange DFs for DC systems in which $(W-$ $\left.E_{\text {ea }}\right)<7 \mathrm{eV}$, i.e., when electron transfer is facile. Such calculations have the potential to widen the scope of existing databases (now only SBH10 ${ }^{22}$ ) of barriers for moleculemetal-surface systems. Indeed, it is likely that the choice of systems in this database ( $\mathrm{SBH} 10$ contains only $\mathrm{H}_{2}$-metal, $\mathrm{CH}_{4}-$ metal, and $\mathrm{N}_{2}-$ metal systems that are all in the greenblue part of the spectrum made up by Figure 1) has led to systematic bias: The inadvertent choice of systems for which GGA-based exchange works well may actually be responsible for the outcome ${ }^{22}$ that the GGA-exchange-based DF performed better than the also tested meta-GGA and hybrid functionals. Even more importantly, the calculations referred to might also increase the range of heterogeneously catalyzed processes that may be simulated reliably based on accurate DFT calculations on the constituent elementary moleculemetal surface reactions.

\section{COMPUTATIONAL METHODS}

For the MS-RPBEl (HSE03-1/3X) DF a $2 \times 2 \mathrm{Al}$ supercell with 4 layers and 15 (10) $\AA$ vacuum distance is used. Furthermore, a plane wave kinetic energy cutoff of 600 (400) $\mathrm{eV}$ and an $8 \times 8 \times 1 \Gamma$-centered $k$-point grid are used. All DFT calculations are performed with the Vienna Ab-initio Simulation Package (VASP version 5.4.4), ${ }^{106-110}$ with a user modification to allow the use of the MS-RPBEl DF, using spin polarization when necessary. The core electrons have been represented with the projector augmented wave (PAW) method. ${ }^{110,111}$ In order to speed up convergence, first-order Methfessel-Paxton smearing ${ }^{12}$ with a width parameter of 0.2 $\mathrm{eV}$ has been employed. Additional information is provided in the Supporting Information.

\section{ASSOCIATED CONTENT}

\section{si Supporting Information}

The Supporting Information is available free of charge at https://pubs.acs.org/doi/10.1021/acs.jpclett.0c02452.

Description of the DFs used (Section S1.1), the DFT calculations (Section S1.2), how the CRP is applied (Section S1.3), the QCT calculations (Section S1.4), and how work function and electron affinity values are obtained (Section S1.5); discussion of several static DFT calculations (Section S2.1), nonself-consistent DFT calculations (Section S2.2), the correlation between
$\left(W-E_{\mathrm{ea}}\right)$ and charge transfer at the TS (Section S2.3), the dependence of $S_{0}$ on the molecular beam conditions (Section S2.4), the alignment of $\mathrm{O}_{2}$ relative to the surface (Section S2.5), and incidence angle (Section $\mathrm{S} 2.6$ ), on $\mathrm{O}_{2}+$ metal systems as benchmarks (Section S3.1), and the development of an SRP functional for $\mathrm{O}_{2}$ $+\mathrm{Al}(111)$ (Section S3.2); elbow plot of the MS-RPBEl and HSE03-1/3X PESs at its minimum barrier geometry (Figures $\mathrm{S} 1$ and $\mathrm{S} 2$, respectively); total magnetic moment of the $\mathrm{fcc} / / 3$ reaction coordinate (Figure S3); $S_{0}$ computed for $(\nu=0, J=0)$ and for $T_{\text {vib }}=300 \mathrm{~K}$, $T_{\text {rot }}=9 \mathrm{~K}$ (Figure S4), and for its dependence on the angle of incidence (Figure S5); work function and electron affinity values (Tables S1 and S2, respectively); effect of varying the screening length parameter and exact exchange fraction (Table S3); vibrational energies (Tables S4 and S5); HSE03-1/3X@RPBE(-vdW-DF1) barrier heights (Tables S6 and S7); excess charge at the TS for several molecule-metal surface systems (Table S8) (PDF)

Software to obtain energies and forces from the MSRPBEl and HSE03-1/3X PESs developed here (ZIP)

\section{AUTHOR INFORMATION}

\section{Corresponding Authors}

Nick Gerrits - Leiden Institute of Chemistry, Leiden University, Gorlaeus Laboratories, 2300 RA Leiden, The Netherlands; 이이.orid/0000-0001-5405-7860;

Email: n.gerrits@lic.leidenuniv.nl

Geert-Jan Kroes - Leiden Institute of Chemistry, Leiden University, Gorlaeus Laboratories, 2300 RA Leiden, The Netherlands; 10 orcid.org/0000-0002-4913-4689; Email: g.j.kroes@chem.leidenuniv.nl

\section{Authors}

Egidius W. F. Smeets - Leiden Institute of Chemistry, Leiden University, Gorlaeus Laboratories, 2300 RA Leiden, The Netherlands; ㅇo이.org/0000-0003-0111-087X

Stefan Vuckovic - Department of Chemistry, University of California, Irvine, California 92697, United States

Andrew D. Powell - Leiden Institute of Chemistry, Leiden University, Gorlaeus Laboratories, 2300 RA Leiden, The Netherlands

Katharina Doblhoff-Dier - Leiden Institute of Chemistry, Leiden University, Gorlaeus Laboratories, 2300 RA Leiden, The Netherlands; (1) orcid.org/0000-0002-5981-9438

Complete contact information is available at:

https://pubs.acs.org/10.1021/acs.jpclett.0c02452

\section{Notes}

The authors declare no competing financial interest.

\section{ACKNOWLEDGMENTS}

This work has been financially supported through a NWO/ CW TOP Grant (No. 715.017.001). Furthermore, this work was carried out on the Dutch national supercomputer with the support of NWO-EW. We thank Jörg Meyer for useful discussions.

\section{REFERENCES}

(1) Peverati, R.; Truhlar, D. G. Quest for a Universal Density Functional: The Accuracy of Density Functionals across a Broad 
Spectrum of Databases in Chemistry and Physics. Philos. Trans. $R$. Soc., A 2014, 372, 20120476.

(2) Mardirossian, N.; Head-Gordon, M. Thirty Years of Density Functional Theory in Computational Chemistry: An Overview and Extensive Assessment of 200 Density Functionals. Mol. Phys. 2017, 115, 2315-2372.

(3) Janesko, B. G. In Density Functionals: Thermochemistry; Johnson, E. R., Ed.; Topics in Current Chemistry; Springer International Publishing: Cham, 2015; Vol. 365; pp 25-51.

(4) Díaz, C.; Pijper, E.; Olsen, R. A.; Busnengo, H. F.; Auerbach, D. J.; Kroes, G. J. Chemically Accurate Simulation of a Prototypical Surface Reaction: $\mathrm{H}_{2}$ Dissociation on $\mathrm{Cu}(111)$. Science 2009, 326, 832-834.

(5) Sementa, L.; Wijzenbroek, M.; van Kolck, B. J.; Somers, M. F.; Al-Halabi, A.; Busnengo, H. F.; Olsen, R. A.; Kroes, G. J.; Rutkowski, M.; Thewes, C.; et al. Reactive Scattering of $\mathrm{H}_{2}$ from $\mathrm{Cu}(100)$ : Comparison of Dynamics Calculations Based on the Specific Reaction Parameter Approach to Density Functional Theory with Experiment. J. Chem. Phys. 2013, 138, 044708.

(6) Wijzenbroek, M.; Kroes, G. J. The Effect of the ExchangeCorrelation Functional on $\mathrm{H}_{2}$ Dissociation on $\mathrm{Ru}(0001)$. J. Chem. Phys. 2014, 140, 084702.

(7) Nattino, F.; Migliorini, D.; Kroes, G.-J.; Dombrowski, E.; High, E. A.; Killelea, D. R.; Utz, A. L. Chemically Accurate Simulation of a Polyatomic Molecule-Metal Surface Reaction. J. Phys. Chem. Lett. 2016, 7, 2402-2406.

(8) Migliorini, D.; Chadwick, H.; Nattino, F.; Gutiérrez-González, A.; Dombrowski, E.; High, E. A.; Guo, H.; Utz, A. L.; Jackson, B.; Beck, R. D.; et al. Surface Reaction Barriometry: Methane Dissociation on Flat and Stepped Transition-Metal Surfaces. J. Phys. Chem. Lett. 2017, 8, 4177-4182.

(9) Ghassemi, E. N.; Wijzenbroek, M.; Somers, M. F.; Kroes, G.-J. Chemically Accurate Simulation of Dissociative Chemisorption of $\mathrm{D}_{2}$ on Pt(111). Chem. Phys. Lett. 2017, 683, 329-335.

(10) Spiering, P.; Shakouri, K.; Behler, J.; Kroes, G.-J.; Meyer, J. Orbital-Dependent Electronic Friction Significantly Affects the Description of Reactive Scattering of $\mathrm{N}_{2}$ from $\mathrm{Ru}(0001)$. J. Phys. Chem. Lett. 2019, 10, 2957-2962.

(11) Ghassemi, E. N.; Smeets, E. W. F.; Somers, M. F.; Kroes, G.-J.; Groot, I. M. N.; Juurlink, L. B. F.; Füchsel, G. Transferability of the Specific Reaction Parameter Density Functional for $\mathrm{H}_{2}+\mathrm{Pt}(111)$ to $\mathrm{H}_{2}+\operatorname{Pt}(211)$. J. Phys. Chem. C 2019, 123, 2973-2986.

(12) Tchakoua, T.; Smeets, E. W. F.; Somers, M.; Kroes, G.-J. Toward a Specific Reaction Parameter Density Functional for $\mathrm{H}_{2}+$ $\mathrm{Ni}(111)$ : Comparison of Theory with Molecular Beam Sticking Experiments. J. Phys. Chem. C 2019, 123, 20420-20433.

(13) Perdew, J. P.; Burke, K.; Ernzerhof, M. Generalized Gradient Approximation Made Simple. Phys. Rev. Lett. 1996, 77, 3865.

(14) Perdew, J. P.; Chevary, J. A.; Vosko, S. H.; Jackson, K. A.; Pederson, M. R.; Singh, D. J.; Fiolhais, C. Atoms, Molecules, Solids, and Surfaces: Applications of the Generalized Gradient Approximation for Exchange and Correlation. Phys. Rev. B: Condens. Matter Mater. Phys. 1992, 46, 6671-6687.

(15) Hammer, B.; Hansen, L. B.; Nørskov, J. K. Improved Adsorption Energetics within Density-Functional Theory Using Revised Perdew-Burke-Ernzerhof Functionals. Phys. Rev. B: Condens. Matter Mater. Phys. 1999, 59, 7413-7421.

(16) Jackson, B.; Nave, S. The Dissociative Chemisorption of Methane on $\mathrm{Ni}(100)$ : Reaction Path Description of Mode-Selective Chemistry. J. Chem. Phys. 2011, 135, 114701.

(17) Jiang, B.; Liu, R.; Li, J.; Xie, D.; Yang, M.; Guo, H. Mode Selectivity in Methane Dissociative Chemisorption on $\mathrm{Ni}(111)$. Chem. Sci. 2013, 4, 3249-3254.

(18) Shen, X.; Zhang, Z.; Zhang, D. H. Communication: Methane Dissociation on $\mathrm{Ni}(111)$ Surface: Importance of Azimuth and Surface Impact Site. J. Chem. Phys. 2016, 144, 101101.

(19) Guo, H.; Jackson, B. Mode-Selective Chemistry on Metal Surfaces: The Dissociative Chemisorption of $\mathrm{CH}_{4}$ on $\mathrm{Pt}(111)$. J. Chem. Phys. 2016, 144, 184709.
(20) Moiraghi, R.; Lozano, A.; Peterson, E.; Utz, A.; Dong, W.; Busnengo, H. F. Nonthermalized Precursor-Mediated Dissociative Chemisorption at High Catalysis Temperatures. J. Phys. Chem. Lett. 2020, 11, 2211-2218.

(21) Wellendorff, J.; Lundgaard, K. T.; Møgelhøj, A.; Petzold, V.; Landis, D. D.; Nørskov, J. K.; Bligaard, T.; Jacobsen, K. W. Density Functionals for Surface Science: Exchange-Correlation Model Development with Bayesian Error Estimation. Phys. Rev. B: Condens. Matter Mater. Phys. 2012, 85, 235149.

(22) Mallikarjun Sharada, S.; Bligaard, T.; Luntz, A. C.; Kroes, G.-J.; Nørskov, J. K. SBH10: A Benchmark Database of Barrier Heights on Transition Metal Surfaces. J. Phys. Chem. C 2017, 121, 19807-19815.

(23) Schimka, L.; Harl, J.; Stroppa, A.; Grüneis, A.; Marsman, M.; Mittendorfer, F.; Kresse, G. Accurate Surface and Adsorption Energies from Many-Body Perturbation Theory. Nat. Mater. 2010, 9, 741-744.

(24) Behler, J.; Delley, B.; Lorenz, S.; Reuter, K.; Scheffler, M. Dissociation of $\mathrm{O}_{2}$ at $\mathrm{Al}(111)$ : The Role of Spin Selection Rules. Phys. Rev. Lett. 2005, 94, 036104.

(25) Füchsel, G.; del Cueto, M.; Díaz, C.; Kroes, G.-J. Enigmatic $\mathrm{HCl}+\mathrm{Au}(111)$ Reaction: A Puzzle for Theory and Experiment. J. Phys. Chem. C 2016, 120, 25760-25779.

(26) Ramos, M.; Díaz, C.; Martínez, A. E.; Busnengo, H. F.; Martín, F. Dissociative and Non-Dissociative Adsorption of $\mathrm{O}_{2}$ on $\mathrm{Cu}(111)$ and $\mathrm{Cu}_{\mathrm{ML}} / \mathrm{Ru}(0001)$ Surfaces: Adiabaticity Takes Over. Phys. Chem. Chem. Phys. 2017, 19, 10217-10221.

(27) Hu, X.; Yang, M.; Xie, D.; Guo, H. Vibrational Enhancement in the Dynamics of Ammonia Dissociative Chemisorption on $\mathrm{Ru}(0001)$. J. Chem. Phys. 2018, 149, 044703.

(28) Füchsel, G.; Zhou, X.; Jiang, B.; Juaristi, J. I.; Alducin, M.; Guo, H.; Kroes, G.-J. Reactive and Nonreactive Scattering of $\mathrm{HCl}$ from $\mathrm{Au}(111)$ : $\mathrm{An} \mathrm{Ab}$ Initio Molecular Dynamics Study. J. Phys. Chem. C 2019, 123, 2287-2299.

(29) Gerrits, N.; Kroes, G.-J. Curious Mechanism of the Dissociative Chemisorption of Ammonia on $\mathrm{Ru}(0001)$. J. Phys. Chem. C 2019, 123, 28291-28300.

(30) Ertl, G. Elementary Steps in Heterogeneous Catalysis. Angew. Chem., Int. Ed. Engl. 1990, 29, 1219-1227.

(31) Sabbe, M. K.; Reyniers, M.-F.; Reuter, K. First-Principles Kinetic Modeling in Heterogeneous Catalysis: An Industrial Perspective on Best-Practice, Gaps and Needs. Catal. Sci. Technol. 2012, 2, 2010-2024.

(32) Wolcott, C. A.; Medford, A. J.; Studt, F.; Campbell, C. T. Degree of Rate Control Approach to Computational Catalyst Screening. J. Catal. 2015, 330, 197-207.

(33) Ertl, G. Primary Steps in Catalytic Synthesis of Ammonia. J. Vac. Sci. Technol., A 1983, 1, 1247-1253.

(34) Rostrup-Nielsen, J. R.; Sehested, J.; Nørskov, J. K. Adv. Catal.; Academic Press, 2002; Vol. 47; pp 65-139.

(35) Perdew, J. P.; Ruzsinszky, A.; Constantin, L. A.; Sun, J.; Csonka, G. I. Some Fundamental Issues in Ground-State Density Functional Theory: A Guide for the Perplexed. J. Chem. Theory Comput. 2009, 5, 902-908.

(36) Libisch, F.; Huang, C.; Liao, P.; Pavone, M.; Carter, E. A. Origin of the Energy Barrier to Chemical Reactions of $\mathrm{O}_{2}$ on $\mathrm{Al}(111)$ : Evidence for Charge Transfer, Not Spin Selection. Phys. Rev. Lett. 2012, 109, 198303.

(37) Zhang, Y.; Yang, W. A Challenge for Density Functionals: SelfInteraction Error Increases for Systems with a Noninteger Number of Electrons. J. Chem. Phys. 1998, 109, 2604-2608.

(38) Cohen, A. J.; Mori-Sánchez, P.; Yang, W. Insights into Current Limitations of Density Functional Theory. Science 2008, 321, 792794.

(39) Perdew, J. P.; Parr, R. G.; Levy, M.; Balduz, J. L. DensityFunctional Theory for Fractional Particle Number: Derivative Discontinuities of the Energy. Phys. Rev. Lett. 1982, 49, 1691-1694.

(40) Li, C.; Zheng, X.; Su, N. Q.; Yang, W. Localized Orbital Scaling Correction for Systematic Elimination of Delocalization Error in Density Functional Approximations. Natl. Sci. Rev. 2018, 5, 203-215. 
(41) Perdew, J. P.; Zunger, A. Self-Interaction Correction to Density-Functional Approximations for Many-Electron Systems. Phys. Rev. B: Condens. Matter Mater. Phys. 1981, 23, 5048-5079.

(42) Kroes, G.-J. Toward a Database of Chemically Accurate Barrier Heights for Reactions of Molecules with Metal Surfaces. J. Phys. Chem. Lett. 2015, 6, 4106-4114.

(43) Behler, J.; Reuter, K.; Scheffler, M. Nonadiabatic Effects in the Dissociation of Oxygen Molecules at the $\mathrm{Al}(111)$ Surface. Phys. Rev. B: Condens. Matter Mater. Phys. 2008, 77, 115421.

(44) Liu, T.; Fu, B.; Zhang, D. H. HCl Dissociating on a Rigid $\mathrm{Au}(111)$ Surface: A Six-Dimensional Quantum Mechanical Study on a New Potential Energy Surface Based on the RPBE Functional. J. Chem. Phys. 2017, 146, 164706.

(45) Liu, Q.; Zhou, X.; Zhou, L.; Zhang, Y.; Luo, X.; Guo, H.; Jiang, B. Constructing High-Dimensional Neural Network Potential Energy Surfaces for Gas-Surface Scattering and Reactions. J. Phys. Chem. C 2018, 122, 1761-1769.

(46) Jiang, B.; Guo, H. Towards an Accurate Specific Reaction Parameter Density Functional for Water Dissociation on $\mathrm{Ni}(111)$ : RPBE versus PW91. Phys. Chem. Chem. Phys. 2016, 18, 2181721824.

(47) Campbell, V. L.; Chen, N.; Guo, H.; Jackson, B.; Utz, A. L. Substrate Vibrations as Promoters of Chemical Reactivity on Metal Surfaces. J. Phys. Chem. A 2015, 119, 12434-12441.

(48) Chadwick, H.; Migliorini, D.; Kroes, G. J. $\mathrm{CHD}_{3}$ Dissociation on Pt(111): A Comparison of the Reaction Dynamics Based on the PBE Functional and on a Specific Reaction Parameter Functional. J. Chem. Phys. 2018, 149, 044701.

(49) Ludwig, J.; Vlachos, D. G. Ab Initio Molecular Dynamics of Hydrogen Dissociation on Metal Surfaces Using Neural Networks and Novelty Sampling. J. Chem. Phys. 2007, 127, 154716.

(50) Golibrzuch, K.; Bartels, N.; Auerbach, D. J.; Wodtke, A. M. The Dynamics of Molecular Interactions and Chemical Reactions at Metal Surfaces: Testing the Foundations of Theory. Annu. Rev. Phys. Chem. 2015, 66, 399-425.

(51) White, J. D.; Chen, J.; Matsiev, D.; Auerbach, D. J.; Wodtke, A. M. Conversion of Large-Amplitude Vibration to Electron Excitation at a Metal Surface. Nature 2005, 433, 503-505.

(52) Nahler, N. H.; White, J. D.; LaRue, J.; Auerbach, D. J.; Wodtke, A. M. Inverse Velocity Dependence of Vibrationally Promoted Electron Emission from a Metal Surface. Science 2008, 321, 11911194.

(53) Bünermann, O.; Jiang, H.; Dorenkamp, Y.; Kandratsenka, A.; Janke, S.; Auerbach, D. J.; Wodtke, A. M. Electron-Hole Pair Excitation Determines the Mechanism of Hydrogen Atom Adsorption. Science 2015, 350, 1346-1349.

(54) Jiang, B.; Alducin, M.; Guo, H. Electron-Hole Pair Effects in Polyatomic Dissociative Chemisorption: Water on Ni(111). J. Phys. Chem. Lett. 2016, 7, 327-331.

(55) Foulkes, W. M. C.; Mitas, L.; Needs, R. J.; Rajagopal, G. Quantum Monte Carlo Simulations of Solids. Rev. Mod. Phys. 2001, $73,33-83$.

(56) Doblhoff-Dier, K.; Meyer, J.; Hoggan, P. E.; Kroes, G.-J. Quantum Monte Carlo Calculations on a Benchmark MoleculeMetal Surface Reaction: $\mathrm{H}_{2}+\mathrm{Cu}(111)$. J. Chem. Theory Comput. 2017, 13, 3208-3219.

(57) Powell, A. D.; Kroes, G. J.; Doblhoff-Dier, K. Quantum Monte Carlo Calculations on Dissociative Chemisorption of $\mathrm{H}_{2}$ on $\mathrm{Al}(110)$ : Minimum Barrier Heights and Their Comparison to DFT Values. J. Chem. Phys. 2020, DOI: 10.1063/5.0022919.

(58) Pozzo, M.; Alfê, D. Hydrogen Dissociation on $\mathrm{Mg}(0001)$ Studied via Quantum Monte Carlo Calculations. Phys. Rev. B: Condens. Matter Mater. Phys. 2008, 78, 245313.

(59) Johnson, R. D., III Computational Chemistry Comparison and Benchmark Database; NIST Standard Reference Database 101. 2020.

(60) Ji, D.-P.; Zhu, Q.; Wang, S.-Q. Detailed First-Principles Studies on Surface Energy and Work Function of Hexagonal Metals. Surf. Sci. 2016, 651, 137-146.
(61) Garron, R. Photoelectricité - Rendement Photoelectriques Des Couches Minces de Magnesium. C.R. Hebd. Seances Acad. Sci. 1964, 258,1458 .

(62) Österlund, L.; Zorić, I.; Kasemo, B. Dissociative Sticking of $\mathrm{O}_{2}$ on $\mathrm{Al}(111)$. Phys. Rev. B: Condens. Matter Mater. Phys. 1997, 55, $15452-15455$.

(63) Yin, R.; Zhang, Y.; Libisch, F.; Carter, E. A.; Guo, H.; Jiang, B. Dissociative Chemisorption of $\mathrm{O}_{2}$ on $\mathrm{Al}(111)$ : Dynamics on a Correlated Wave-Function-Based Potential Energy Surface. J. Phys. Chem. Lett. 2018, 9, 3271-3277.

(64) Kurahashi, M.; Yamauchi, Y. Steric Effect in $\mathrm{O}_{2}$ Sticking on $\mathrm{Al}(111)$ : Preference for Parallel Geometry. Phys. Rev. Lett. 2013, 110, 246102.

(65) Katz, G.; Kosloff, R.; Zeiri, Y. Abstractive Dissociation of Oxygen over $\mathrm{Al}(111)$ : A Nonadiabatic Quantum Model. J. Chem. Phys. 2004, 120, 3931-3948.

(66) Mosch, C.; Koukounas, C.; Bacalis, N.; Metropoulos, A.; Gross, A.; Mavridis, A. Interaction of Dioxygen with $\mathrm{Al}$ Clusters and Al(111): A Comparative Theoretical Study. J. Phys. Chem. C 2008, $112,6924-6932$.

(67) Livshits, E.; Baer, R.; Kosloff, R. Deleterious Effects of LongRange Self-Repulsion on the Density Functional Description of $\mathrm{O}_{2}$ Sticking on Aluminum. J. Phys. Chem. A 2009, 113, 7521-7527.

(68) Bacalis, N. C.; Metropoulos, A.; Gross, A. Theoretical Study of the $\mathrm{O}_{2}$ Interaction with a Tetrahedral $\mathrm{Al}_{4}$ Cluster. J. Phys. Chem. A 2010, 114, 11746-11750.

(69) Liu, H.-R.; Xiang, H.; Gong, X. G. First Principles Study of Adsorption of $\mathrm{O}_{2}$ on Al Surface with Hybrid Functionals. J. Chem. Phys. 2011, 135, 214702.

(70) Cheng, J.; Libisch, F.; Carter, E. A. Dissociative Adsorption of $\mathrm{O}_{2}$ on $\mathrm{Al}(111)$ : The Role of Orientational Degrees of Freedom. $J$. Phys. Chem. Lett. 2015, 6, 1661-1665.

(71) Paranthaman, S.; Moon, J.; Hong, K.; Kim, J.; Kim, D. E.; Kim, J.; Kim, T. K. Reactivity of Molecular Oxygen with Aluminum Clusters: Density Functional and Ab Initio Molecular Dynamics Simulation Study. Int. J. Quantum Chem. 2016, 116, 547-554.

(72) Martin-Gondre, L.; Crespos, C.; Larrégaray, P.; Rayez, J. C.; Conte, D.; van Ootegem, B. Detailed Description of the Flexible Periodic London-Eyring-Polanyi-Sato Potential Energy Function. Chem. Phys. 2010, 367, 136-147.

(73) Karplus, M.; Porter, R. N.; Sharma, R. D. Exchange Reactions with Activation Energy. I. Simple Barrier Potential for $\left(\mathrm{H}, \mathrm{H}_{2}\right)$. J. Chem. Phys. 1965, 43, 3259-3287.

(74) Smeets, E. W. F.; Voss, J.; Kroes, G.-J. Specific Reaction Parameter Density Functional Based on the Meta-Generalized Gradient Approximation: Application to $\mathrm{H}_{2}+\mathrm{Cu}(111)$ and $\mathrm{H}_{2}+$ $\mathrm{Ag}(111)$. J. Phys. Chem. A 2019, 123, 5395-5406.

(75) Heyd, J.; Scuseria, G. E.; Ernzerhof, M. Hybrid Functionals Based on a Screened Coulomb Potential. J. Chem. Phys. 2003, 118, $8207-8215$

(76) Heyd, J.; Scuseria, G. E.; Ernzerhof, M. Erratum: "Hybrid Functionals Based on a Screened Coulomb Potential" [J. Chem. Phys. 118, 8207 (2003)]. J. Chem. Phys. 2006, 124, 219906.

(77) Sun, J.; Xiao, B.; Fang, Y.; Haunschild, R.; Hao, P.; Ruzsinszky, A.; Csonka, G. I.; Scuseria, G. E.; Perdew, J. P. Density Functionals That Recognize Covalent, Metallic, and Weak Bonds. Phys. Rev. Lett. 2013, 111, 106401.

(78) Cortona, P. Note: Theoretical Mixing Coefficients for Hybrid Functionals. J. Chem. Phys. 2012, 136, 086101.

(79) Guido, C. A.; Brémond, E.; Adamo, C.; Cortona, P. Communication: One Third: A New Recipe for the PBE0 Paradigm. J. Chem. Phys. 2013, 138, 021104.

(80) Zhao, Y.; Truhlar, D. G. Density Functional Theory for Reaction Energies: Test of Meta and Hybrid Meta Functionals, Range-Separated Functionals, and Other High-Performance Functionals. J. Chem. Theory Comput. 2011, 7, 669-676.

(81) Brémond, É.; Pérez-Jiménez, Á. J.; Sancho-García, J. C.; Adamo, C. Range-Separated Hybrid Density Functionals Made Simple. J. Chem. Phys. 2019, 150, 201102. 
(82) Moltved, K. A.; Kepp, K. P. The Metal Hydride Problem of Computational Chemistry: Origins and Consequences. J. Phys. Chem. A 2019, 123, 2888-2900.

(83) Zhao, Q.; Kulik, H. J. Stable Surfaces That Bind Too Tightly: Can Range-Separated Hybrids or DFT $+\mathrm{U}$ Improve Paradoxical Descriptions of Surface Chemistry? J. Phys. Chem. Lett. 2019, 10, 5090-5098.

(84) Laikov, D. N. Simple Exchange Hole Models for Long-RangeCorrected Density Functionals. J. Chem. Phys. 2019, 151, 094106.

(85) Jana, S.; Patra, A.; Constantin, L. A.; Samal, P. Screened RangeSeparated Hybrid by Balancing the Compact and Slowly Varying Density Regimes: Satisfaction of Local Density Linear Response. J. Chem. Phys. 2020, 152, 044111.

(86) Busnengo, H. F.; Salin, A.; Dong, W. Representation of the 6D Potential Energy Surface for a Diatomic Molecule near a Solid Surface. J. Chem. Phys. 2000, 112, 7641-7651.

(87) Olsen, R. A.; Busnengo, H. F.; Salin, A.; Somers, M. F.; Kroes, G. J.; Baerends, E. J. Constructing Accurate Potential Energy Surfaces for a Diatomic Molecule Interacting with a Solid Surface: $\mathrm{H}_{2}+\mathrm{Pt}(111)$ and $\mathrm{H}_{2}+\mathrm{Cu}(100)$. J. Chem. Phys. 2002, 116, 3841-3855.

(88) Porter, R. N.; Raff, L. M. In Dynamics of Molecular Collisions: Part B; Miller, W. H., Ed.; Modern Theoretical Chemistry; Springer US: Boston, MA, 1976; pp 1-52.

(89) Karikorpi, M.; Holloway, S.; Henriksen, N.; Nørskov, J. K. Dynamics of Molecule-Surface Interactions. Surf. Sci. 1987, 179, L41-L48.

(90) Binetti, M.; Weiße, O.; Hasselbrink, E.; Katz, G.; Kosloff, R.; Zeiri, Y. The Role of Nonadiabatic Pathways and Molecular Rotations in the Oxygen Abstraction Reaction on the $\mathrm{Al}(111)$ Surface. Chem. Phys. Lett. 2003, 373, 366-371.

(91) Tiwari, A. K.; Nave, S.; Jackson, B. The Temperature Dependence of Methane Dissociation on $\mathrm{Ni}(111)$ and $\mathrm{Pt}(111)$ : Mixed Quantum-Classical Studies of the Lattice Response. J. Chem. Phys. 2010, 132, 134702.

(92) Tiwari, A. K.; Nave, S.; Jackson, B. Methane Dissociation on Ni(111): A New Understanding of the Lattice Effect. Phys. Rev. Lett. 2009, 103, 253201.

(93) Mori-Sánchez, P.; Cohen, A. J.; Yang, W. Many-Electron SelfInteraction Error in Approximate Density Functionals. J. Chem. Phys. 2006, 125, 201102.

(94) Mori-Sánchez, P.; Cohen, A. J.; Yang, W. Localization and Delocalization Errors in Density Functional Theory and Implications for Band-Gap Prediction. Phys. Rev. Lett. 2008, 100, 146401.

(95) Bao, J. L.; Gagliardi, L.; Truhlar, D. G. Self-Interaction Error in Density Functional Theory: An Appraisal. J. Phys. Chem. Lett. 2018, 9, 2353-2358.

(96) Bartlett, R. J. Adventures in DFT by a Wavefunction Theorist. J. Chem. Phys. 2019, 151, 160901.

(97) Berland, K.; Jiao, Y.; Lee, J.-H.; Rangel, T.; Neaton, J. B.; Hyldgaard, P. Assessment of Two Hybrid van Der Waals Density Functionals for Covalent and Non-Covalent Binding of Molecules. J. Chem. Phys. 2017, 146, 234106.

(98) Jiao, Y.; Schröder, E.; Hyldgaard, P. Extent of Fock-Exchange Mixing for a Hybrid van Der Waals Density Functional? J. Chem. Phys. 2018, 148, 194115.

(99) Marom, N.; Tkatchenko, A.; Rossi, M.; Gobre, V. V.; Hod, O.; Scheffler, M.; Kronik, L. Dispersion Interactions with DensityFunctional Theory: Benchmarking Semiempirical and Interatomic Pairwise Corrected Density Functionals. J. Chem. Theory Comput. 2011, 7, 3944-3951.

(100) Lonsdale, D. R.; Goerigk, L. The One-Electron SelfInteraction Error in 74 Density Functional Approximations: A Case Study on Hydrogenic Mono- and Dinuclear Systems. Phys. Chem. Chem. Phys. 2020, 22, 15805-15830.

(101) Kim, M.-C.; Sim, E.; Burke, K. Understanding and Reducing Errors in Density Functional Calculations. Phys. Rev. Lett. 2013, 111, 073003.
(102) Vuckovic, S.; Song, S.; Kozlowski, J.; Sim, E.; Burke, K. Density Functional Analysis: The Theory of Density-Corrected DFT. J. Chem. Theory Comput. 2019, 15, 6636-6646.

(103) Song, S.; Sim, E.; Vuckovic, S.; Burke, K. How Errors in Densities Contaminate Empirical Density Functionals. arXiv 2020, 2008.01261.

(104) Skelton, J. M.; Gunn, D. S. D.; Metz, S.; Parker, S. C. Accuracy of Hybrid Functionals with Non-Self-Consistent Kohn-Sham Orbitals for Predicting the Properties of Semiconductors. J. Chem. Theory Comput. 2020, 16, 3543-3557.

(105) Patra, A.; Peng, H.; Sun, J.; Perdew, J. P. Rethinking CO Adsorption on Transition-Metal Surfaces: Effect of Density-Driven Self-Interaction Errors. Phys. Rev. B: Condens. Matter Mater. Phys. 2019, 100, 035442.

(106) Kresse, G.; Hafner, J. Ab Initio Molecular-Dynamics Simulation of the Liquid-Metal-Amorphous-Semiconductor Transition in Germanium. Phys. Rev. B: Condens. Matter Mater. Phys. 1994, 49, $14251-14269$.

(107) Kresse, G.; Hafner, J. Ab Initio Molecular Dynamics for Liquid Metals. Phys. Rev. B: Condens. Matter Mater. Phys. 1993, 47, $558-561$.

(108) Kresse, G.; Furthmüller, J. Efficient Iterative Schemes for Ab Initio Total-Energy Calculations Using a Plane-Wave Basis Set. Phys. Rev. B: Condens. Matter Mater. Phys. 1996, 54, 11169-11186.

(109) Kresse, G.; Furthmüller, J. Efficiency of Ab-Initio Total Energy Calculations for Metals and Semiconductors Using a Plane-Wave Basis Set. Comput. Mater. Sci. 1996, 6, 15-50.

(110) Kresse, G.; Joubert, D. From Ultrasoft Pseudopotentials to the Projector Augmented-Wave Method. Phys. Rev. B: Condens. Matter Mater. Phys. 1999, 59, 1758-1775.

(111) Blöchl, P. E. Projector Augmented-Wave Method. Phys. Rev. B: Condens. Matter Mater. Phys. 1994, 50, 17953-17979.

(112) Methfessel, M.; Paxton, A. T. High-Precision Sampling for Brillouin-Zone Integration in Metals. Phys. Rev. B: Condens. Matter Mater. Phys. 1989, 40, 3616-3621. 